\section{Distribuição dos recursos humanos em oito hospitais gerais de São Paulo ${ }^{1}$}

\author{
Olímpio J. Nogueira V. Bittar ${ }^{2}$
}

\section{RESUMO}

O desenvolvimento tecnológico e o aparecimento de novas especialidades médicas motivaram um aumento na divisão de trabalho nos hospitais, com conseqüente criação de subáreas. A redistribuição dos recursos humanos nos hospitais passa, então, a ser uma meta importante para o alcance da eficiência. Através de entrevistas realizadas entre maio e outubro de 1993, com diretores e superintendentes de oito hospitais em São Paulo, Brasil, o presente estudo descreveu e analisou a distribuição dos recursos humanos em quatro grandes áreas: infra-estrutura; internação clínico-cirúrgica; área complementar de diagnóstico e terapêutica; ambulatório e emergência. O estudo analisou também a proporção de funcionários por leito, além de traçar uma comparação entre hospitais públicos e privados. Os resultados revelaram que, em média, a relação funcionários/leito é igual a 6,8. A distribuição percentual de funcionários por grandes áreas, conforme o estudo, foi de 46,9\% na infra-estrutura, 10,7\% na área complementar de diagnóstico e terapêutica, 32,0\% na internação e 10,3\% na área de ambulatório/emergência.

1 Extraído da tese de livre-docência intitulada “Produtividade em hospitais", apresentada ao Departamento de Prática de Saúde Pública da Universidade de São Paulo em novembro de 1994.

2 Instituto Dante Pazzanese de Cardiologia, Avenida Dr. Dante Pazzanese, 500, CEP 04012-180, São Paulo, SP, Brasil (email: bittar@usp.br). Correspondência e pedidos de separatas devem ser enviados a esse endereço.
Há algumas décadas, o número de funcionários por leito é utilizado por hospitais em todo o mundo como parâmetro para estimar tanto a necessidade de pessoal em unidades a serem planejadas ou organizadas quanto a necessidade de ampliações de unidades existentes (1). Nesse contexto, funcionários são definidos como todos os profissionais empregados pelo hospital, inclusive terceiros; leitos são todas as camas à disposição de pacientes, inclusive nas unidades de terapia intensiva (excluindo camas destinadas a pacientes em observação).

A partir da década de 70, graças ao desenvolvimento tecnológico, uma grande proporção das atividades hospitalares passou a ser realizada fora do leito, ou seja, no ambulatório. Por exemplo, atualmente, entre 20 e $40 \%$ das cirurgias podem ser realizadas em ambulatório (2). Dessa forma, é preciso estabelecer novos parâmetros para estimar a necessidade de mão-de-obra. $\mathrm{O}$ desenvolvimento tecnológico propiciou também a utilização maior e melhor da área complementar de diagnóstico e terapêutica, que passou a exigir mais recursos humanos. Esse fato tornou ainda mais inviável a utilização somente da relação funcionários por leito para o planejamento e gestão das unidades.

$\mathrm{O}$ aumento no número de especialidades médicas também influenciou a relação funcionários/leito e a diversificação da utilização do ambulatório e das unidades de internação. No Brasil, o Conselho Federal de Medicina (3) reconhece 65 especialidades; nos Estados Unidos, esse número chegava a 85 em 1990 (4). Cada especialidade gera um desenvolvimento tecnológico próprio, que inclui novos equipamentos, procedimentos e drogas e requer mão-de-obra especial, muitas vezes intensiva.

A partir dessas mudanças, pode-se observar um aumento da relação entre o número de funcionários por leito ao longo do tempo, bem como uma total discrepância entre os valores encontrados em diversos países, ou em diversos hospitais no Brasil. No Japão, essa relação varia, em alguns hospitais, de 1 a 1,4 (1). Nos Estados Unidos, a relação entre número de funcionários e leitos aumentou de 1,48 em 1946 para 4,13 em $1984 .^{3}$ Ainda nesse país, Michel et al. (5) sugerem que a média de

\footnotetext{
3 Azevedo AC. "Contribuição à metodologia de análise de hospitais complexos" [tese de livre-docência]. São Paulo: Faculdade de Saúde Pública da Universidade de São Paulo; 1989.
} 
empregados por paciente, na indústria hospitalar como um todo, gira em torno de 3; em hospitais com fins lucrativos a média é de 2,5. No México, o índice de funcionários por leito chega a 3,5 (6) para hospitais de 144 leitos com atendimento em nível primário e secundário e serviços de diagnóstico e terapias não-complexos.

Um estudo realizado em 26 hospitais complexos (que realizam, por exemplo, cirurgia cardiovascular e oncológica) no Brasil encontrou uma variação de 1 a 7,2 na proporção de funcionários por leitos. ${ }^{4}$ De acordo com dados coletados em hospitais públicos de São Paulo (1), a relação entre funcionários e leitos varia de 4 a 9, lembrando-se que muitas vezes não são contabilizados os serviços contratados a terceiros, como limpeza, manutenção e laboratório, entre outros. Esse trabalho (1) discute também o estudo da coordenadoria de assistência hospitalar da Secretaria de Estado da Saúde de São Paulo, que estabeleceu um quadro de pessoal para seus hospitais gerais, onde a relação funcionário/ leito varia de 2,9 a 3,9 .

A redefinição dos parâmetros para o planejamento e gestão das unidades hospitalares têm conseqüências importantes para a quantificação, qualificação, treinamento e desenvolvimento de recursos humanos, bem como na sua distribuição pelas diversas áreas hospitalares. Contudo, existem poucos dados disponíveis sobre esses aspectos, tanto em âmbito nacional como em âmbito internacional. Embora um primeiro trabalho sobre dimensionamento de pessoal para hospitais de 25 a 100 leitos tenha sido publicado por Shafer (7) em 1949, pouco se sabe a respeito da distribuição atual da força de trabalho nos hospitais, embora este fator esteja implicitamente ligado ao desempenho dos hospitais, não só em termos da quantidade de pacientes que podem ser atendidos, mas, principalmente, da qualidade do atendimento.

O objetivo principal desta pesquisa é, portanto, descrever a distribuição dos recursos humanos, especialmente do pessoal de enfermagem, em oito hospitais gerais do Município de São Paulo. Além disso, o estudo compara a relação entre funcionários e leitos nos hospitais públicos e privados investigados.

\section{MATERIAIS E MÉTODOS}

A pesquisa descrita no presente trabalho foi desenvolvida em oito hospitais gerais do Município de São Paulo. Seis dos hospitais investigados eram

\footnotetext{
4 Ver nota de pé de página No. 3.
}

privados e dois, públicos. Todos são considerados de grande porte (entre 151 e 500 leitos). Os hospitais prestam assistência nos níveis secundário e terciário e foram escolhidos por serem representantes típicos de hospitais públicos e privados. ${ }^{5}$

Para a caracterização dos hospitais, informações foram levantadas acerca de índices de morbidade; faixa etária da população atendida; especialidades médicas; corpo clínico (aberto ou fechado, isto é, se o hospital permite ou não que, além do seu próprio corpo clínico, outros médicos utilizem as instalações para prestar assistência a doentes); existência no próprio hospital de áreas geradoras de demanda e áreas de influência na resolubilidade, ou seja, áreas que facilitam e agilizam os diagnósticos e terapêuticas (como ambulatório, emergência e serviços complementares de diagnóstico e terapêutica, inclusive com utilização de tecnologia de ponta como angioplastia, procedimentos laparoscópicos, litotripsia e outras); existência de áreas de cuidados intensivos ou de observação, além de áreas de suporte (como recuperação pós-operatória, planejamento de suprimento, eletrônica, marketing e custos). Finalmente, foram levados em conta a localização do hospital, principalmente para demandas de ambulatório e emergência, o tipo de clientela (particular, convênios ou Sistema Único de Saúde) e a capacidade operacional (8), ou seja, o número de leitos efetivamente existentes no hospital, respeitada a legislação em vigor.

Os hospitais foram identificados com letras de $\mathrm{A}$ a $\mathrm{H}$, sendo que os dois hospitais públicos (um hospital municipal e um hospital universitário), receberam as letras A e $\mathrm{B}$, respectivamente. Os demais, privados, foram sorteados, sendo caracterizados com as letras C-H. Nas descrições dos resultados e discussão, a citação dos hospitais se dará pelas siglas HA-HH. A distribuição dos leitos por hospital na época do estudo era a seguinte: HA, 193 leitos; HB, 224; HC, 336; HD, 151; HE, 127; HF, 185; HG, 150 e HH, 145 leitos.

Os hospitais, caracterizados a partir dos elementos listados acima, foram divididos em dois grupos: o primeiro grupo é composto por dois hospitais públicos e dois privados, que guardam características semelhantes no que diz respeito à morbidade, especialidades médicas, tecnologia utilizada, corpo clínico (fechado) e número e tipo de serviços complementares de diagnóstico e terapêutica. Este grupo é composto pelos hospitais HA, HB, HE e HF. A população atendida por esses hospitais é restrita e distribuída da seguinte forma: a população que

\footnotetext{
Bittar OJNV. "Produtividade em hospitais" [tese de livre docência] São Paulo: Faculdade de Saúde Pública da Universidade de São Paulo; 1994.
} 
utiliza o HA é composta por funcionários públicos municipais; o HB atende funcionários da Universidade de São Paulo e a população residente no bairro de Butantã; o HE atende pessoas pertencentes a um determinado sindicato; o HF recebe pessoas filiadas a uma determinada medicina de grupo.

O segundo grupo, HC, HD, HG e HH é composto somente por hospitais privados cujo corpo clínico é aberto (HC e HH também possuem médicos empregados). Esses hospitais possuem recursos tecnológicos avançados (nível terciário) e um maior número de serviços complementares de diagnóstico e terapêutica. A população atendida é coberta por seguro saúde, medicina de grupo, cooperativa médica ou particular.

O primeiro contato com os hospitais ocorreu no mês de maio de 1993, quando o projeto foi apresentado e discutido com a direção de cada hospital. A partir daí, seis visitas foram realizadas, nas quais os superintendentes e os diretores dos hospitais foram entrevistados. Uma das visitas foi dedicada exclusivamente a conhecer as dependências físicas do hospital.

Nas outras visitas, através de questionários estruturados, procurou-se obter dados e documentos que permitissem conhecer em detalhes a organização, administração e produção das unidades. A coleta de dados terminou em 15 de outubro de 1993.

Ênfase especial foi dada à política de recursos humanos, com abordagem das áreas de recrutamento e seleção, administração de pessoal, administração de cargos e salários, administração de benefícios, treinamento e desenvolvimento, indicadores de absenteísmo, rotatividade da mão-de-obra e percentuais dos gastos com folha de pagamentos, benefícios, encargos, área de engenharia de segurança e medicina do trabalho, além de existência de Comissão Interna de Prevenção de Acidentes (CIPA). A CIPA foi incluída por estar relacionada ao bom desempenho do trabalho dentro do hospital, prevenindo doenças profissionais e acidentes de trabalho.

Como apenas o primeiro grupo de hospitais apresentava corpo clínico fechado e como mesmo neste grupo havia grandes diferenças de carga horária, turnos e plantões entre os médicos, a apresentação da distribuição dos recursos humanos excluiu a área médica. Além disso, como um profissional presta serviços em várias áreas, torna-se difícil caracterizar se seu tempo de trabalho é dedicado mais ao ambulatório, emergência ou internação.

Os recursos humanos foram divididos em quatro grandes áreas: infra-estrutura; internação clínicocirúrgica; área complementar de diagnóstico e terapêutica; ambulatório e emergência. $\mathrm{Na}$ área de infra-estrutura foram incluídas as seguintes subáreas: administração de gabinete, administração de recursos humanos, armazenamento, artes gráficas, assistência técnica eletrônica, atendimento ao cliente, auditoria/convênios, biblioteca, centro de material esterilizado, centro de técnica de administração de materiais e medicamentos, comercial, compras, comissão técnica de engenharia e arquitetura, contabilidade, controladoria, creche, crédito/cobrança, custo/orçamento, engenharia, engenharia de segurança e medicina do trabalho, expediente, farmácia, farmácia semi-industrial, importação, informática, jurídico, lanchonete, lavanderia/costura, limpeza, manutenção, marketing, material, nutrição, oficina impressora, planejamento, serviço de arquivo médico e estatísticas (SAME), superintendência, tesouraria, vigilância e zeladoria. Propositadamente, incluiu-se nesta área a subárea de esterilização de material, classificada também como atividade de infra-estrutura.

Fazem parte da área de internação clínicocirúrgica os funcionários de todas as subáreas em que o paciente permanece internado em leitos ou camas e é submetido a procedimentos invasivos e não-invasivos, bem como os funcionários das áreas de apoio. As seguintes subáreas foram incluídas nessa área: anestesia, apoio à anestesia, centro cirúrgico, centro obstétrico, centro de recuperação anestésica, clínica pediátrica, infecção hospitalar, internação clínico-cirúrgica, internação clínica obstétrica, unidade neonatal, unidade de moléstia infecciosa, UTI adulto e UTI pediátrica. O pessoal dedicado à gerência de enfermagem e os responsáveis pela educação continuada em enfermagem estão aqui fixados.

$\mathrm{Na}$ área complementar de diagnóstico e terapêutica foram incluídos análises clínicas, anatomia patológica, angiografia/hemodinâmica, banco de sangue, banco de tecidos, betaterapia, biologia molecular, cardiotocografia, centro de reforço e informação oncológica, dermatologia, diálise peritonial, ecocardiografia, endoscopia, eletrocardiografia, eletroencefalografia, eletromiografia, ergometria, fisioterapia, fonoaudiologia, hemodiálise, holter, litotripsia, medicina nuclear, microondoterapia prostática, neurofisiologia, oftalmologia, ortóptica, pneumonologia, potencial evocado, quimioterapia, radiologia, reprodução humana, radioterapia, reabilitação, ressonância magnética, tomografia, ultra-sonografia e urodinâmica.

Finalmente, em ambulatório e emergência foram incluídos ambulatório propriamente dito, ambulatório de pediatria assistencial, cirurgia ambulatorial, centro de recuperação anestésica ambulatorial, revisão de saúde, serviço social e psicologia.

A equipe de enfermagem encontra-se presente em todas as áreas e mereceu destaque na presente análise. $\mathrm{O}$ pessoal de enfermagem foi dividido nas seguintes funções: enfermeiro; técnico; auxiliar de enfermagem; atendente; técnico 
assistencial; técnico de material; auxiliar de produção; operador de autoclave.

Para todas as grandes áreas e subáreas foi solicitado o número de profissionais por categoria, carga horária, tipos de turnos e plantões, número de horas trabalhadas por dia e o regime jurídico do contrato de trabalho. Informações a respeito de serviços de terceiros foram levantadas com relação à área de atuação, quantificação e qualificação dos profissionais, horas trabalhadas e tipo de contrato. Os dados foram analisados através de estatísticas descritivas, como amplitude de variação, proporções e médias, que poderão servir de parâmetro para outros estudos.

\section{RESULTADOS}

A distribuição dos recursos humanos pelas quatro grandes áreas nos oito hospitais analisados encontra-se na tabela 1. A área de infra-estrutura, que não atende diretamente ao paciente, mas sim a outras áreas do hospital, compreende em torno de $47 \%$ (amplitude de 3,7\% a 50,7\%) dos recursos humanos dos hospitais. Na área de internação clínico-cirúrgica, a proporção de pessoal variou de $18,1 \%$ no HA a $43,5 \%$ no HF, sendo que a média dos oito hospitais foi de $32,0 \%$. As subáreas da internação clínico cirúrgica são similares em todos os hospitais, com exceção da subárea de apoio à anestesia, que somente é encontrada no HA, HB e HC.

A coleta e tabulação dos dados na área complementar de diagnóstico e terapêutica é mais difícil porque o mesmo pessoal pode ser utilizado em uma ou mais subáreas. Além disso, o pessoal de internação e ambulatório também pode ser utilizado para procedimentos realizados com menor freqüência e que não justifiquem a alocação permanente de funcionários. As maiores proporções de funcionários alocados nesta área foram observadas no $\mathrm{HH}$, com $16,3 \%$; $\mathrm{HD}$, com $14,8 \%$; $\mathrm{HC}$, com $14,6 \%$; e $H G$, com $11,3 \%$. No primeiro grupo, a amplitude encontrada foi de $2,7 \%$ (no HF) a $9,1 \%$ (no HE). A média dos oito hospitais foi de 10,7\%. $\mathrm{Na}$ área de ambulatório/emergência, a proporção média de funcionários foi de 10,3\%, com uma amplitude de 5,7 a 22,7\%.

Quanto à equipe de enfermagem, presente nas quatro grandes áreas analisadas, os dados revelaram que o pessoal de enfermagem representa 39\% dos recursos humanos do total das quatro áreas, com uma amplitude que variou de 31,4 a $54,5 \%$. A tabela 2 apresenta a distribuição do pessoal de enfermagem por categoria em cada um dos hospitais. Ao todo, 3922 profissionais estão empregados nos setores de enfermagem dos oito hospitais analisados. Metade desses profissionais $(50,5 \%)$ são auxiliares de enfermagem. Entre os enfermeiros, foram encontrados profissionais com as seguintes especializações (em nível de pós graduação lato sensu): geral, clínico-cirúrgica, obstetrícia, pediatria, terapia intensiva, pronto-socorro, clínica médica, cirurgia ambulatorial, endoscopia, oncologia e serviço auxiliar de diagnóstico.

Quanto aos técnicos em enfermagem, alguns hospitais (HA, HB, HC e HH) não tinham funcionários nessa categoria. Os auxiliares de produção e os operadores de autoclave foram encontrados somente no $\mathrm{HF}$ e $\mathrm{HH}$, respectivamente.

A terceirização dos serviços hospitalares, definida como um processo de transferência de funções para outras empresas que possam executá-las (9), ocorre de maneira diversa em cada hospital. A manutenção de equipamentos de alta complexidade

TABELA 1. Distribuição absoluta e percentual dos recursos humanos nos hospitais participantes por grandes áreas e relação funcionários/leito, São Paulo, Brasil, 1993

\begin{tabular}{|c|c|c|c|c|c|c|c|c|c|}
\hline \multirow[b]{3}{*}{ Áreas } & \multicolumn{9}{|c|}{ Hospitais } \\
\hline & A & B & $E$ & $\mathrm{~F}$ & C & $\mathrm{D}$ & $\mathrm{G}$ & $\mathrm{H}$ & \\
\hline & $\%$ & $\%$ & $\%$ & $\%$ & $\%$ & $\%$ & $\%$ & $\%$ & Média \\
\hline \multirow[t]{2}{*}{ Infra-estrutura } & 1218 & 662 & 242 & 406 & 1094 & 374 & 391 & 630 & - \\
\hline & 50,7 & 45,5 & 45,3 & 47,9 & 49,2 & 37,6 & 48,7 & 50,3 & 46,9 \\
\hline \multirow{4}{*}{$\begin{array}{l}\text { Complementar de } \\
\text { diagnóstico e terapêutica } \\
\text { Internação }\end{array}$} & 200 & 125 & 49 & 23 & 324 & 147 & 91 & 204 & - \\
\hline & 8,3 & 8,6 & 9,1 & 2,7 & 14,6 & 14,8 & 11,3 & 16,3 & 10,7 \\
\hline & 435 & 452 & 206 & 369 & 677 & 354 & 237 & 364 & - \\
\hline & 18,1 & 31,1 & 38,6 & 43,5 & 30,4 & 35,6 & 29,5 & 29,1 & 32,0 \\
\hline \multirow[t]{2}{*}{ Ambulatório/emergência } & 546 & 213 & 37 & 50 & 128 & 118 & 83 & 53 & - \\
\hline & 22,7 & 14,7 & 6,9 & 5,9 & 5,7 & 11,9 & 10,3 & 4,2 & 10,3 \\
\hline \multirow[t]{2}{*}{ Total } & 2399 & 1452 & 534 & 848 & 2223 & 993 & 802 & 1251 & - \\
\hline & 100 & 100 & 100 & 100 & 100 & 100 & 100 & 100 & 100 \\
\hline Funcionários ${ }^{a}$ /leito & 12,4 & 5,9 & 4,2 & 4,6 & 6,6 & 6,6 & 5,3 & 8,6 & 6,8 \\
\hline
\end{tabular}

a Não inclui médicos e dentistas. 
TABELA 2. Distribuição da equipe de enfermagem dos hospitais gerais por categoria profissional, São Paulo, Brasil, 1993

\begin{tabular}{|c|c|c|c|c|c|c|c|c|c|}
\hline \multirow[b]{2}{*}{ Equipe de enfermagem } & \multicolumn{9}{|c|}{ Hospitais } \\
\hline & $\begin{array}{l}A \\
\%\end{array}$ & $\begin{array}{l}\text { B } \\
\%\end{array}$ & $\begin{array}{l}E \\
\%\end{array}$ & $\begin{array}{l}\mathrm{F} \\
\%\end{array}$ & $\begin{array}{l}C \\
\%\end{array}$ & $\begin{array}{l}D \\
\%\end{array}$ & $\begin{array}{l}\mathrm{G} \\
\%\end{array}$ & $\begin{array}{l}\mathrm{H} \\
\%\end{array}$ & $\begin{array}{c}\text { Total } \\
\%\end{array}$ \\
\hline Enfermeiro & $\begin{array}{r}107 \\
14,2\end{array}$ & $\begin{array}{r}164 \\
26,2\end{array}$ & $\begin{array}{r}39 \\
16,5\end{array}$ & $\begin{array}{r}80 \\
17,3\end{array}$ & $\begin{array}{r}162 \\
21,1\end{array}$ & $\begin{array}{r}81 \\
20,4\end{array}$ & $\begin{array}{r}48 \\
17,2\end{array}$ & $\begin{array}{r}76 \\
18,9\end{array}$ & $\begin{array}{r}757 \\
19,3\end{array}$ \\
\hline Técnico & - & - & $\begin{array}{r}3 \\
1,3\end{array}$ & $\begin{array}{r}24 \\
5,2\end{array}$ & - & $\begin{array}{r}36 \\
9,1\end{array}$ & $\begin{array}{r}9 \\
3,2\end{array}$ & - & $\begin{array}{r}72 \\
1,8\end{array}$ \\
\hline Auxiliar de enfermagem & $\begin{array}{r}366 \\
48,6\end{array}$ & $\begin{array}{r}180 \\
28,8\end{array}$ & $\begin{array}{r}100 \\
42,2\end{array}$ & $\begin{array}{r}279 \\
60,4\end{array}$ & $\begin{array}{r}433 \\
56,5\end{array}$ & $\begin{array}{r}228 \\
57,4\end{array}$ & $\begin{array}{r}153 \\
54,8\end{array}$ & $\begin{array}{r}241 \\
60,0\end{array}$ & $\begin{array}{r}1980 \\
50,5\end{array}$ \\
\hline Atendente & $\begin{array}{r}280 \\
37,2\end{array}$ & $\begin{array}{r}245 \\
39,1\end{array}$ & $\begin{array}{r}95 \\
40,1\end{array}$ & $\begin{array}{r}42 \\
9,1\end{array}$ & $\begin{array}{r}171 \\
22,3\end{array}$ & $\begin{array}{r}52 \\
13,1\end{array}$ & $\begin{array}{r}69 \\
24,7\end{array}$ & $\begin{array}{r}83 \\
20,6\end{array}$ & $\begin{array}{r}1037 \\
26,4\end{array}$ \\
\hline Técnico assistencial & - & $\begin{array}{r}22 \\
3,5\end{array}$ & - & $\begin{array}{r}3 \\
0,6\end{array}$ & - & - & - & - & $\begin{array}{r}25 \\
0,6\end{array}$ \\
\hline Técnico de material & - & $\begin{array}{r}15 \\
2,4\end{array}$ & - & $\begin{array}{r}11 \\
2,4\end{array}$ & - & - & - & - & $\begin{array}{r}26 \\
0,7\end{array}$ \\
\hline Auxiliar de produção & - & - & - & $\begin{array}{r}23 \\
5,0\end{array}$ & - & - & - & - & $\begin{array}{r}23 \\
0,6\end{array}$ \\
\hline Operador de autoclave & - & - & - & - & - & - & - & $\begin{array}{r}2 \\
0,5\end{array}$ & $\begin{array}{r}2 \\
0,1\end{array}$ \\
\hline Total & $\begin{array}{l}753 \\
100\end{array}$ & $\begin{array}{l}626 \\
100\end{array}$ & $\begin{array}{l}237 \\
100\end{array}$ & $\begin{array}{l}462 \\
100\end{array}$ & $\begin{array}{l}766 \\
100\end{array}$ & $\begin{array}{l}397 \\
100\end{array}$ & $\begin{array}{l}279 \\
100\end{array}$ & $\begin{array}{l}402 \\
100\end{array}$ & $\begin{array}{r}3922 \\
100\end{array}$ \\
\hline
\end{tabular}

apresenta-se terceirizada em todos. Além disso, no HA a limpeza encontra-se terceirizada; no HD e HF são terceirizadas todas as subáreas complementares de diagnóstico (como por exemplo, banco de sangue, laboratório clínico e anatomia patológica); no $\mathrm{HH}$ a anatomia patológica e o banco de sangue são terceirizados. No HG as únicas áreas não terceirizadas são a enfermagem, SAME , contabilidade, finanças e superintendência. Todos os hospitais tinham CIPA, com exceção do HB, que se utiliza da comissão existente na Universidade de São Paulo.

\section{DISCUSSÃO}

O primeiro fato realçado quando se analisa a tabela 1 diz respeito à participação percentual da área de infra-estrutura. Com exceção do HD, que apresentou percentual menor $(37,6 \%)$, todos os outros hospitais são próximos, embora a composição das subáreas tenha variado entre os hospitais (dados não mostrados).

Na seqüência dessa análise, um outro fato diz respeito à distribuição dos recursos humanos entre as áreas, afetada por particularidades criadas por fatores externos e pelos próprios hospitais no que diz respeito à demanda, tecnologia utilizada, especialidades médicas e localização do hospital, que facilita ou dificulta os atendimentos de emergência. Por exemplo, a localização do hospital muitas vezes dificulta a contratação de pessoal. Os funcionários preferem hospitais centrais, servidos por ônibus ou por condução mais fácil e de baixo custo. O mesmo acontece com os pacientes. A tecnologia, por sua vez, facilita ou dificulta a contratação de mão de obra na medida em que existe ou não a possibilidade de treinamento local.

Na comparação de hospitais públicos e privados, chama a atenção a concentração de pessoal na área de ambulatório/emergência dos hospitais públicos (tabela 1). Esse fato é perfeitamente aceitável tendo em vista a universalidade das ações de saúde preconizadas pelo Sistema Único de Saúde (SUS) adotado no Brasil, em que existe uma integração do atendimento de saúde nos níveis federal, estadual e municipal, sendo que o município deveria assumir as ações de saúde.

Em relação ao grande número de subáreas da área de infra-estrutura, que concentram quase 50\% dos recursos humanos nos hospitais analisados, embora o aumento no número de pessoal utilizado possa ser interpretado como uma diminuição na produtividade, estudos (10) demonstram que esse aumento, ao contrário, pode gerar lucros. Isto se aplica também a outras áreas, onde a criação de um número maior de subáreas com diferentes graus de complexidade, grande número de profissionais de saúde e, especificamente, de médicos, pode melhorar a quantidade e qualidade do atendimento.

Com relação à quantificação dos recursos humanos, observa-se que existem categorias padrão (por exemplo, enfermeiro, escriturário, servente), no aspecto funcional, que são utilizadas em todos os hospitais. Contudo, há hospitais que se 
utilizam da mão-de-obra com alguma especialidade de atuação (por exemplo, engenheiro de produção e estatístico), objetivando o desenvolvimento de determinadas áreas relacionadas à melhoria das condições de atendimento do hospital. Em relação à política de recursos humanos, verifica-se que nos hospitais privados há maior autonomia no recrutamento e seleção, na administração de salários, benefícios, treinamento e desenvolvimento (dados não mostrados). Estes fatores se refletem diretamente na quantidade e distribuição do pessoal e no aumento de produtividade dessas unidades, em razão da colocação do profissional adequado na sua função e da utilização de mecanismos mais flexíveis de motivação, como políticas de cargos e salários e de benefícios, definidas e adequadas ao mercado, além de maiores investimentos em treinamento e desenvolvimento de pessoal. Em alguns hospitais (HC, HF, HD), nota-se uma preocupação especial com planejamento, marketing e outras atividades que não são comumente encontradas na rede hospitalar (dados não mostrados).

\section{Índice de funcionários/leito}

A análise desta relação passa pelo conhecimento da organização dos hospitais, das áreas e subáreas de ambulatório/emergência e de diagnóstico e terapia colocadas à disposição dos pacientes e médicos e também internamente, daquelas áreas que servem de apoio para as atividades administrativas e técnicas.

À primeira vista, a análise deste indicador mostra que, no primeiro grupo, os hospitais privados, HE e HF, apresentam uma menor relação entre funcionários e leitos. Por conseqüência, estes hospitais se destacam como os que melhor aproveitamento fazem de sua mão-de-obra.

No segundo grupo, onde estão colocados os hospitais de alta complexidade, a relação funcionários/leito, sem levar em consideração o corpo clínico, é basicamente a mesma entre os hospitais HC e HD $(6,6)$, embora o HC apresente um número maior de áreas de suporte, de cuidados intensivos e geradoras de demanda e influência na resolubilidade. No HG, esta relação é ainda mais baixa $(5,3)$. É possível que este resultado seja decorrência do alto grau de terceirização de serviços. $\mathrm{O} \mathrm{HH}$, por sua vez, apresenta o índice mais elevado $(8,6)$, que pode ser justificado pela clientela, caracterizada como de elevada faixa etária e também pela patologia predominante de neoplasias.

\section{Equipe de enfermagem}

A área de enfermagem é uma das áreas em que o dimensionamento de pessoal é tido como importante para que se obtenha resultados na produtividade (11). Dado que a equipe de enfermagem encontra-se presente nas quatro grandes áreas apresentadas nesse trabalho, resolveu-se apresentála com maiores detalhes. Uma primeira observação na tabela 2 é a de que, além do enfermeiro, do técnico, do auxiliar e do atendente de enfermagem, alguns hospitais agregam em seu quadro técnico assistencial o técnico de material, o auxiliar de produção e o operador de autoclave, estes dois últimos exclusivamente lotados no centro de esterilização de material.

Um estudo (12) com 11 hospitais em Ribeirão Preto, Estado de São Paulo, encontrou para um grupo de enfermagem composto por 2813 profissionais a seguinte relação: $11,23 \%$ de enfermeiros; $7,67 \%$ de técnicos; $38,02 \%$ de auxiliares; e $43,80 \%$ de atendentes, o que mostra uma melhor qualificação dos profissionais nos oito hospitais pesquisados no presente estudo (em 3922 profissionais, 19,3\% de enfermeiros; $3,1 \%$ de técnicos; $50,5 \%$ de auxiliares de enfermagem; $26,4 \%$ de atendentes de enfermagem; e $0,7 \%$ de outros auxiliares e operadores).

\section{CONCLUSÕES}

A dependência dos hospitais em relação à força de trabalho para o desenvolvimento de suas atividades vem aumentando ao longo das últimas décadas, em decorrência da complexidade que acompanha a tecnologia. São raros os estudos que detalham a quantificação e distribuição dos recursos humanos, nas diversas áreas e subáreas existentes nestas unidades, o que dificulta o estabelecimento de parâmetros para o planejamento, gestão e avaliação das mesmas.

Os hospitais privados apresentam uma distribuição melhor de funcionários/leito do que os hospitais públicos. Esta relação aumenta à medida em que o hospital dispõe de maior quantidade de subáreas no ambulatório e na área completar de diagnóstico e terapêutica, bem como no atendimento de casos mais graves e de maior faixa etária.

Conclui-se pela necessidade de estudos mais aprofundados sobre recursos humanos em hospitais, para que se obtenham parâmetros que possibilitem melhorar a eficiência do sistema de saúde. 


\section{REFERÊNCIAS}

1. Bittar OJNV. Dimensionamento de pessoal na área hospitalar. Prev Dados 1992; 7(1):10-18.

2. Bittar OJNV. Ambulatório: da simplicidade à complexidade é uma solução. Rev Paul Hosp 1990;37(1/4):8-13.

3. Conselho Federal de Medicina. Resolução no. 1441/94, 12 de agosto de 1994. Diário Oficial da União, Brasília, 16 de agosto de 1994.

4. Bittar OJNV. Especialidades médicas no Brasil. Prev Dados 1991;6(1):31-42.

5. Michel A, Skaked I, Daley J. The proprietary hospital industry: a financial analysis 1972-1982. Soc Sci Med 1985; 21(3):235-242.

6. Soberón G, Chávez MR, Ortega-Lomelín R, Martínez-Narváez G，Ruiz-lbarra I. Los nuevos hospitales del programa de reconstrucción y reordenamiento de los servicios de salud en el área metropolitana. Salud Publ Mex 1989;31(1): 91-99.

7. Shafer MK. Staffing the general hospital 25 to 100 beds. Illinois: American Hospital Association; 1949.

8. Brasil, Ministério da Saúde. Normas e padrões de construções e instalações de serviços de saúde: conceitos e definições hospital geral de pequeno e médio portes, unidades sanitárias. Brasília: Ministério da Saúde; 1978.

9. Brasil HG. A empresa e a estratégia da terceirização. Rev Admin Emp 1993;33 (2):6-11.

10. Serway GD, Strum DW, Haug WF. Alternative indications for measuring hospital productivity. Hosp Health Serv Admin 1987; 32(3):379-398.
11. Harju M, Sabatino F. Productivity efforts on the rise. Hospitals 1984; nov:89-90.

12. Lana FCF, Mello D, Gir E, Nogueira MS, Hildebrand JM. Características da rede hospitalar de Ribeirão Preto: categoria de financiamento, cobertura populacional e recursos humanos. Medicina (Ribeirão Preto) 1993;26(3):408-418.

Manuscrito aceito para publicação em 12 de setembro de 1996

ABSTRACT Technological development and the appearance of new medical specialties have led to increased division of labor in hospitals, with the resultant creation of subareas. Therefore, the redistribution of human resources in

Distribution of human resources in eight general hospitals in São Paulo hospitals has become an important aim in order to achieve efficiency. On the basis of interviews conducted between May and October 1993 with the directors and superintendents of eight hospitals in São Paulo, Brazil, this study describes and analyzes the distribution of human resources in four broad areas: infrastructure, clinico-surgical inpatient care, the complementary area of diagnostics and therapeutics, and emergency and outpatient care. The study also analyzed the ratio of employees per bed, besides outlining a comparison of public and private hospitals. The results revealed that, on average, the ratio of employees to beds was 6.8. The proportional distribution of employees by broad areas was found to be $46.9 \%$ in infrastructure, $10.7 \%$ in diagnostics and therapeutics, $32.0 \%$ in inpatient care, and $10.3 \%$ in outpatient/emergency care. 